\title{
A computational investigation on cavitating flow characteristic of innovative nozzle structures in diesel engine
}

\author{
Jiarui $\mathrm{Li}^{1}$, Bo Yuan ${ }^{1,2,3, a}$, Liangming $\mathrm{Pan}^{1,3}$ and Shuai $\mathrm{Jia}^{1}$ \\ ${ }^{1}$ Key Laboratory of Low-grade Energy Utilization Technologies and Systems, Chongqing \\ University,Chongqing University,Chongqing,400044,China \\ ${ }^{2}$ State Key Laboratory of Coal Mine Disaster Dynamics and Control,Chongqing \\ University, Chongqing,400044,China \\ ${ }^{3}$ Postdoctoral Research Station of Power Engineering \& Engineering Thermophysics, Chongqing \\ University, Chongqing,400044,China \\ aboyuanyuan@cqu.edu.cn
}

\begin{abstract}
Keywords: innovative nozzle structures; cavitating flow; angle of inclination; numerical simulation Abstract: The relative differences in the flow characteristics of the fuel within nozzle holes of diesel injectors significantly influences the atomization and combustion characteristics of diesel engines. The numerical simulation of cavitating flow developed fully in nozzle holes of a vertical four hole injector with mixture homogeneous equilibrium model was carried out. And then the effects of four essential parameters (the use of innovative nozzle construction, elliptical geometry nozzle hole, the ratio of nozzle's length to orifice's diameter and hole inclination angle) on the cavitating flow in nozzle holes were studied. It is finally concluded that the medium expansion nozzle is better than that of the ordinary nozzle and the three-stage tapered nozzle and elliptical geometry with vertically oriented major axis are less prone to cavitate and the cavitation is more likely to occur when the nozzle mounting angle is smaller.
\end{abstract}

\section{Introduction}

With the rapid development of society, energy and environmental problems have become the most important problems affecting the future survival of mankind. The research on the energy saving and emission reduction of diesel engine is more and more urgent Since the full implementation of the national IV emission standards. Fuel injector is the most complexcore part of the fuel injection system, which plays important role in fuel injection and its control process. The fuel temperature, pressure, fuel injection, turbulent kinetic energy and so will further affect the fuel atomization and the subsequent combustion quality when it enters the combustion chamber from the nozzle. The control of the internal flow of the diesel engine nozzle is the key to achieving high efficiency combustion,which is important for energy saving and decontamination. The high pressure flow of fuel in the confined space of the nozzle is prone to cavitation. Steam bubbles quickly rupture in the higher pressure area, which will make the metal surface to withstand the impact, And then the nozzle will be cavitation wear.On the other hand, cavitation can enhance atomization quality and improve combustion efficiency ${ }^{[1-3]}$. Recent studies have found that the turbulence near the wall surface in the cavitation area of the nozzle is significantly enhanced when cavitation occurs ${ }^{[4]}$. It is generally believed that this phenomenon is due to the fact that part of the pressure fluctuation caused by cavitation bubble collapse is converted into turbulent kinetic energy. The turbulence intensity affects the stability of the entire flow and therefore has an effect on the initial atomization formation. This also explains the phenomenon of reduced crushing length and Jet cone angle increases ${ }^{[5]}$.

In recent years and the next few years, one of the key breakthroughs in the energy saving and emission reduction of diesel engines is the study of the mechanism of cavitation. This research has been carried out at home and abroad. He Z X et al. ${ }^{[6]}$ Studied the effect of jet pressure on the flow characteristics of the proportional injection orifice. Its results show that the hole area increases with the injection pressure increasing continuously toward the downstream of the nozzle. Zuo-Yu Sun et al. ${ }^{[7]}$ asserted that as the nozzle wall roughness increases, the mass flow and flow coefficient increase first 
and then decrease, and the appropriate roughness can control cavitation in a certain degree. Wang et al. ${ }^{[8]}$ studied the effects of fuel temperature on the inside cavitation nozzle. He et al. ${ }^{[9-12]}$ analyzed the critical point of cavitation and hydraulic plunger flow and the flow energy loss during the process of hydraulic plunger flow. Jiang et al. ${ }^{[13]}$ Compared the flow and near field spray in the transparent injector of the nozzle which is the actual size nozzle with under different fuels. The flow condition inside the nozzle and the cavitation are not only related to the injection pressure, the back pressure, the fuel temperature, the nozzle wall roughness, but also the geometry of the injector. Therefore, The method of computer numerical simulation(CFD) was used to study the influence of the nozzle geometry, the nozzle injection angle, the length of the nozzle and so on on the internal cavity flow in the nozzle.

\section{Simulation method}

\section{Nozzle structures}

The cavities in the four-hole vertical injector were analyzed by ANSYS Fluent. The needle injector has four nozzles and evenly distributed, the nozzle diameter is $0.3 \mathrm{~mm}$, the nozzle length is $1 \mathrm{~mm}$, the needle diameter is $4 \mathrm{~mm}$, the needle front end is $3 \mathrm{~mm}$, the needle valve is sealed with the vertebral angle of $60^{\circ}$, Needle valve body seal $59^{\circ}$, pressure chamber length $1 \mathrm{~mm}$. The nozzle hole is a sharp edge transition.

As follows is the comparison of the nozzle geometry model.

The first group of programs is to simulate three different nozzle structure of the four-hole vertical injection nozzle flow, as shown in Figure 1. Figure hole size are shows in Figure 1.

Tab 1 Hole size

\begin{tabular}{clllllll}
\hline & $\mathrm{D}$ & $\mathrm{L}$ & $\mathrm{L} 1$ & $\mathrm{~L} 2$ & $\mathrm{~L} 3$ & $\mathrm{D} 1$ & $\mathrm{D} 2$ \\
\hline length/[mm] & 0.3 & 1 & 0.3 & 0.3 & 0.2 & 0.5 & 0.4 \\
\hline
\end{tabular}

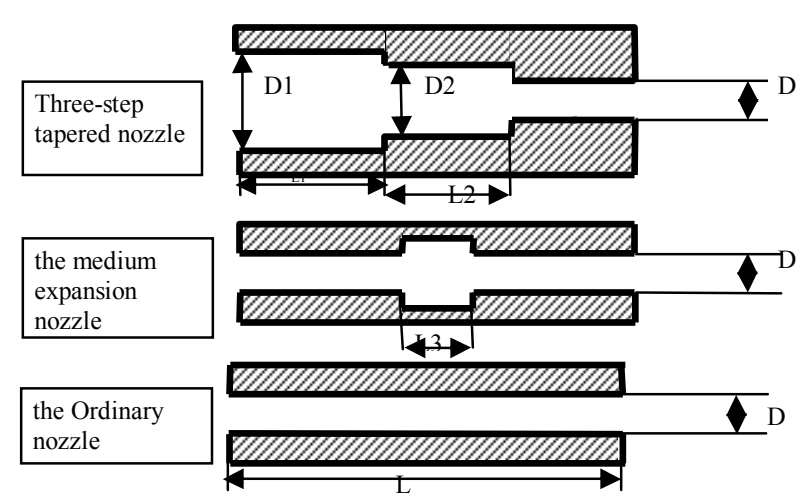

Fig. 1 nozzle structure
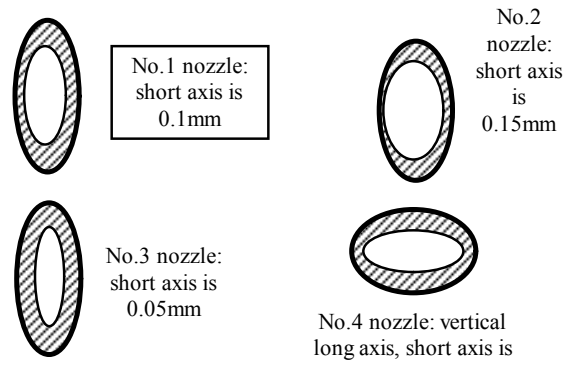

Fig. 2 oval nozzle

The second group of programs is to study the flow of elliptical nozzle, as shown in Figure 2. The long axis of elliptical nozzle is $0.2 \mathrm{~mm}$ and the minor axis is $0.05 \mathrm{~mm}, 0.1 \mathrm{~mm}$, and $0.15 \mathrm{~mm}$. The short axis of the vertical long axis elliptical nozzle is $0.15 \mathrm{~mm}$.

The third set of schemes is to analyze the flow conditions in circular nozzles of different aspect ratios, as shown in Table 2.

Tab 2 Circular nozzle length to diameter ratio distribution

\begin{tabular}{lllll}
\hline Group & 1 & 2 & 3 & 4 \\
\hline Nozzle length $/[\mathrm{mm}]$ & 1 & 1 & 1 & 0.5 \\
Hole diameter $/[\mathrm{mm}]$ & 0.2 & 0.3 & 0.4 & 0.3 \\
Aspect ratio & 5 & $10 / 3$ & $5 / 2$ & $5 / 3$ \\
\hline
\end{tabular}

The fourth group of programs studied the flow conditions of circular nozzles at different installation angles, as shown in Table 3. 
Tab 3 The inclination angle of the round nozzle

\begin{tabular}{llll}
\hline Group & 5 & 6 & 7 \\
\hline Tilt angle $/\left[^{\circ}\right]$ & 30 & 45 & 60 \\
\hline
\end{tabular}

\section{Physical model and numerical method}

The numerical simulation model uses a mixture homogeneous equilibrium model, which is used to calculate Cavitation. Numerical simulation of gas - liquid two - phase turbulence in the cavity flow is carried out. The basic control equations include continuity equations and momentum equations. Because the Realizable $\mathrm{k}-\varepsilon$ model uses a new turbulent viscosity formula, it is possible to more accurately simulate the diffusion velocity of a circular jet, And it is the best turbulence model in the separation flow calculation and the secondary flow calculation, The turbulence is modeled using the Realizable $\mathrm{k}-\varepsilon$ model. The wall is treated with an enhanced wall function. Zwart-Gerber-Belamri model and Schnerr \& Sauer model are more robust than Singhal cavitation model, and compared with the Zwart-Gerber-Belamri and Schnerr \& Sauer cavitation models, the Singhal model is more stable and easy to converge, And the Singhal model is more sensitive to back pressure ${ }^{[14]}$. In addition, the Zwart-Gerber-Belamri model has higher prediction accuracy under the large flow coefficient ${ }^{[15]}$. The simulation assumes that the flow is isothermal and does not take into account the relative motion between the gas and liquid phases. The basic equations of the cavitation model are given in detail in document ${ }^{[14]}$. Import and export are used pressure boundary, due to the emergence of holes within the hole, the nozzle outlet pressure is often different from the cylinder pressure. The exit boundary is extended to the cylinder and the calculation area shown in Fig. 3 is established. The inlet pressure is 80 $\mathrm{MPa}$ and the outlet pressure is $0.1 \mathrm{MPa}$. On the solid wall boundary, there is no wall jet or suction, so the flow on the solid wall surface without slip and ignore the heat transfer. The pressure - velocity correlation algorithm is chosen with the coupled algorithm, the pressure equation is PRESTO, and the other equations adopt the first - order upwind scheme. The FLUENT is used to solve the three dimensional numerical simulation without time - dependent steady - state, and the finite volume method based on the inner node is adopted for the dispersion of the equation. The cavitation condition of the internal fluid in the nozzle is mainly discussed, and the internal fluid channel of the injector is structured, which is shown in Figure 3. The nozzle part of the grid is locally encrypted; the number of meshes is 2.37 million.

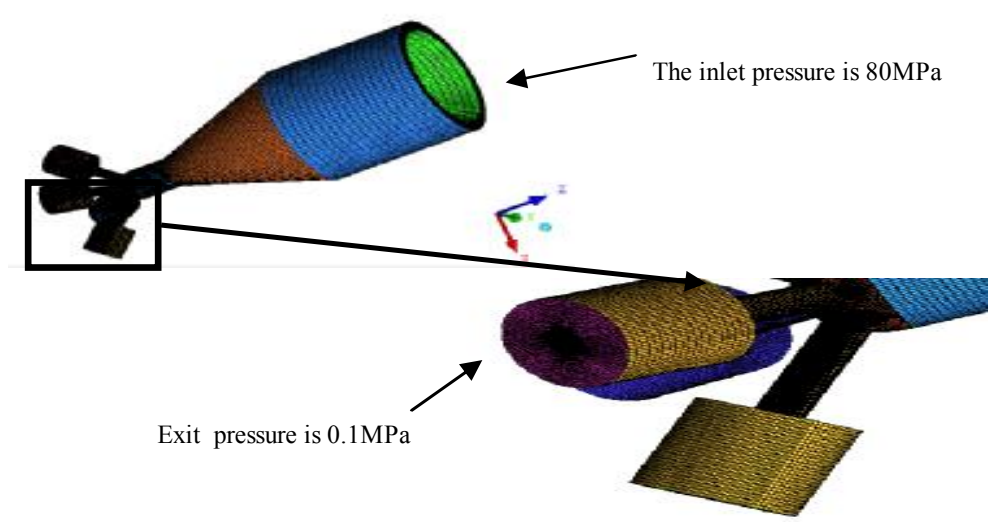

Fig. 3 Meshing scheme

\section{Results and discussion}

\section{Effects of Nozzle structure within control valve on flow characteristics}

The nozzle structure consists of a Ordinary nozzle, a three-stage tapered nozzle to a medium-expansion nozzle, and the effect of the structure on the flow is different. When the needle valve lift is $0.25 \mathrm{~mm}$, the nozzle of the three different structures Numerical simulation of comparative analysis. Figure 4, Figure 5, Figure 6, respectively, shows the three structural nozzle hole distribution, velocity, pressure numerical results. 
It can be seen from Figure 4 that the inner cavity of the three-stage tapered nozzle is thinnest; the internal pressure drop of the three-stage tapered nozzle is not very obvious compared to the other two nozzles. The main reason is that the nozzle diameter at the inlet is larger than that of the other two nozzles. In addition, when the inner diameter of the third section is reduced to $0.3 \mathrm{~mm}$, there is no obvious cavitation, because the progressive increase in the diameter of the fluid from the large space into the small space pressure drop. In addition, the hole in the medium-expansion nozzle is slightly thicker than the ordinary nozzle. Then, since the inner diameter of the middle portion becomes larger, the continuation extension of the cavitation is cut off. In this way, the flow disorder caused by cavitation in the three-stage tapered nozzle is the weakest, the atomization effect is also the worst. For the expansion of the nozzle will not reduce the turbulence intensity, but also can effectively control the cavitation layer extension length.

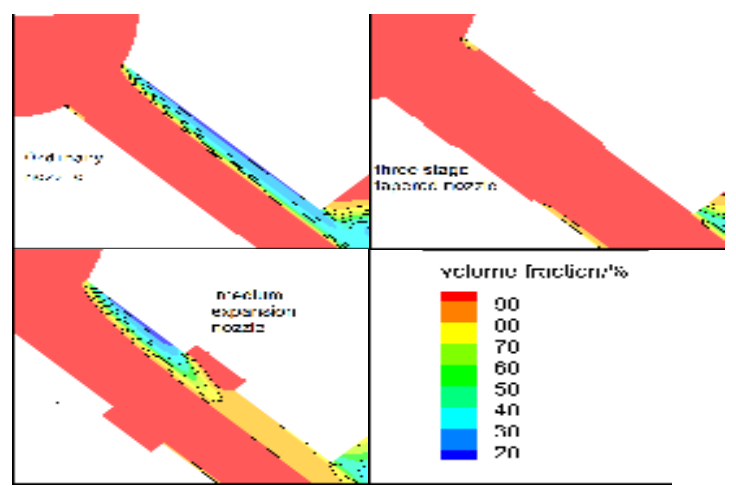

Fig. 4 Vapour mass fraction at different structure nozzles

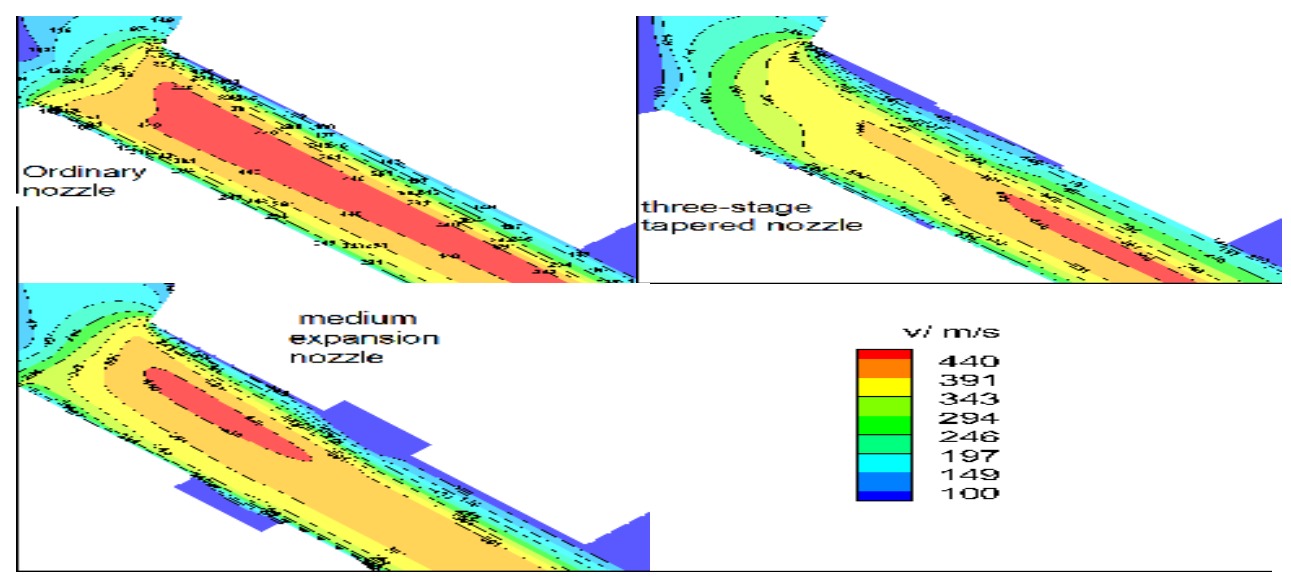

Fig. 5 Different structure nozzle velocity distribution

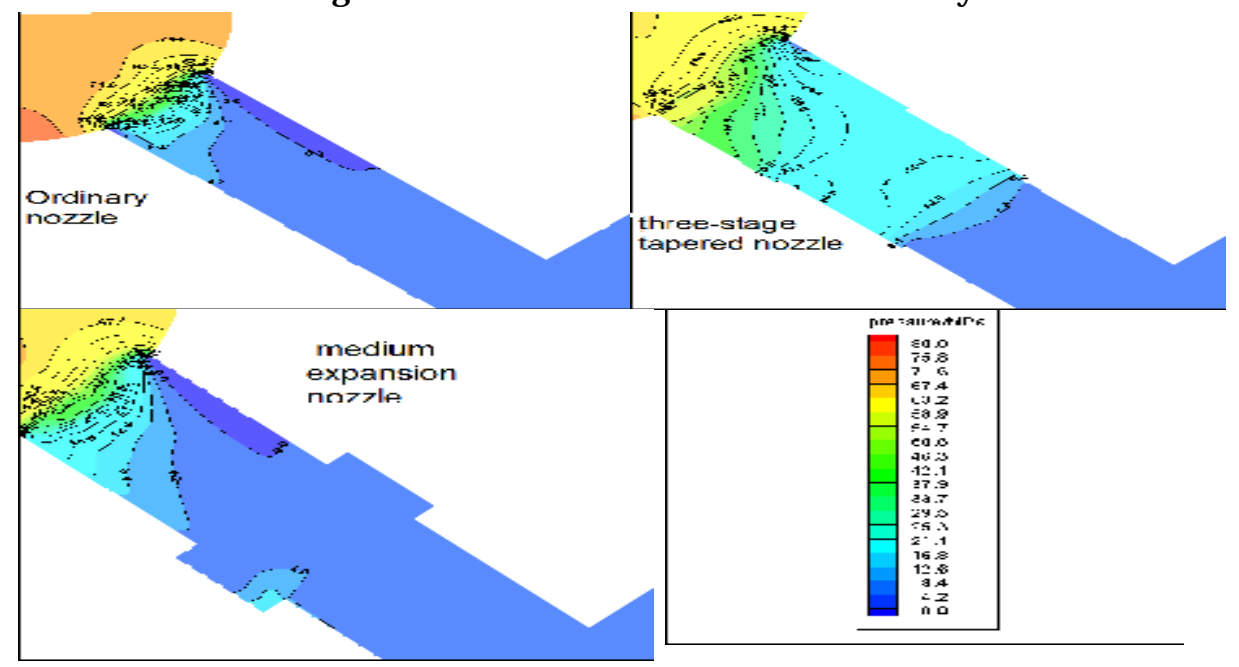

Fig. 6 Different structure nozzle pressure distribution 
As can be seen from Fig. 5, the velocity at the inlet of the three-stage tapered nozzle is the lowest, due to the relative relaxation of the fluid into the micro-space in the three-stage tapered nozzle compared to the other two nozzles. The Liquid flow disorder of the nozzle outlet caused by turbulence in the Ordinary nozzle and the medium-expansion nozzle is more severe than that in the three-stage tapered nozzle. From this point of view, the maximum amount of fuel injection, the ordinary nozzle and the expansion of the nozzle to produce oil mist droplets to be thinner. The speed of the fuel in the medium - expansion nozzle is slightly lower than that of the ordinary nozzle, but its velocity gradient is larger and the turbulence can be slightly larger than that of the ordinary nozzle. It can be seen that the pressure distribution of the three-stage tapered nozzles is most moderate. The medium-expansion nozzles still have pressure fluctuations at the rear end of the nozzles compared to the ordinary nozzles. The same shows that the fuel turbulence intensity is weakest in the three-stage tapered nozzle, and it is the strongest in the Medium expansion nozzle. All in all, from this point of view, the cavitation of the medium-expansion nozzle is more easily controlled and its atomization characteristics are optimal.

Effects of the elliptical nozzle within control valve on flow characteristics

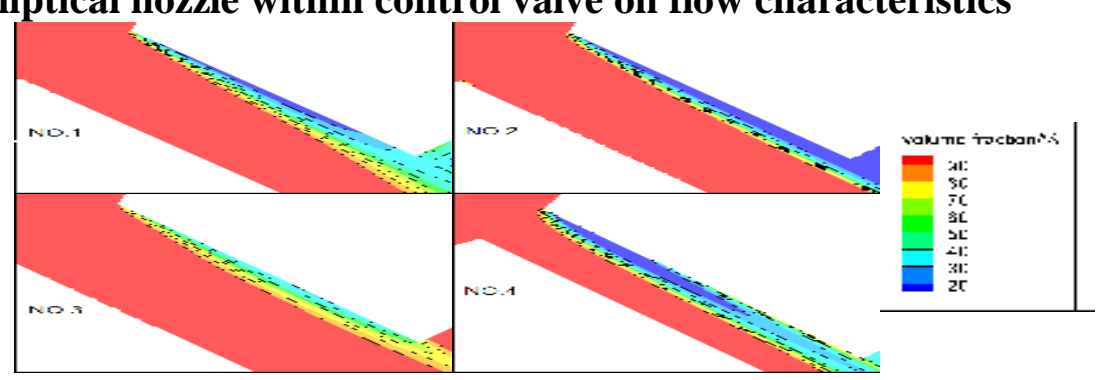

Fig. 7 Vapour mass fraction at oval nozzle

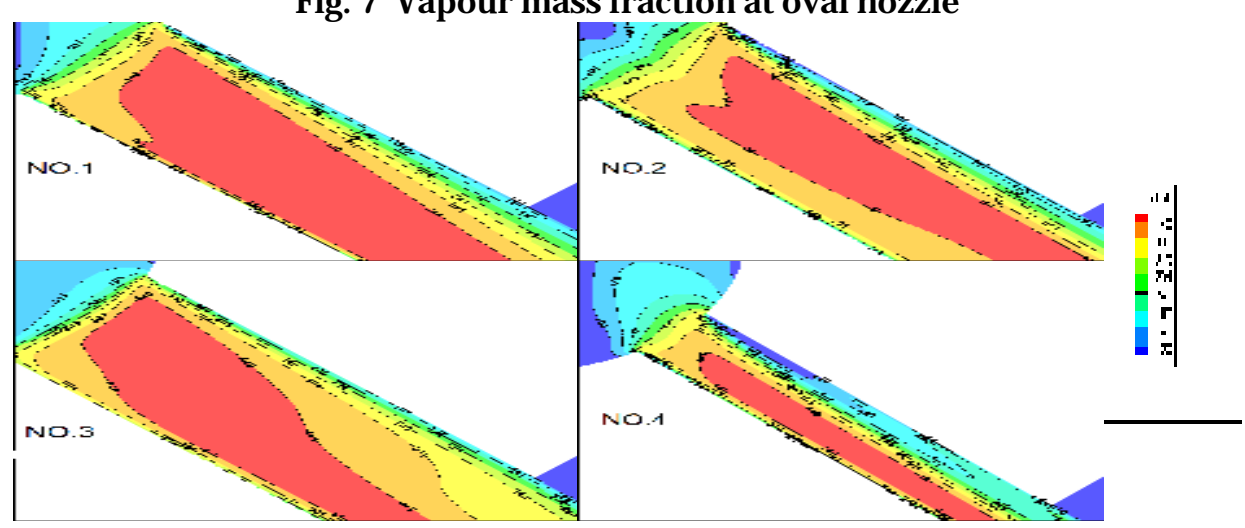

Fig. 8 Oval nozzle velocity distribution

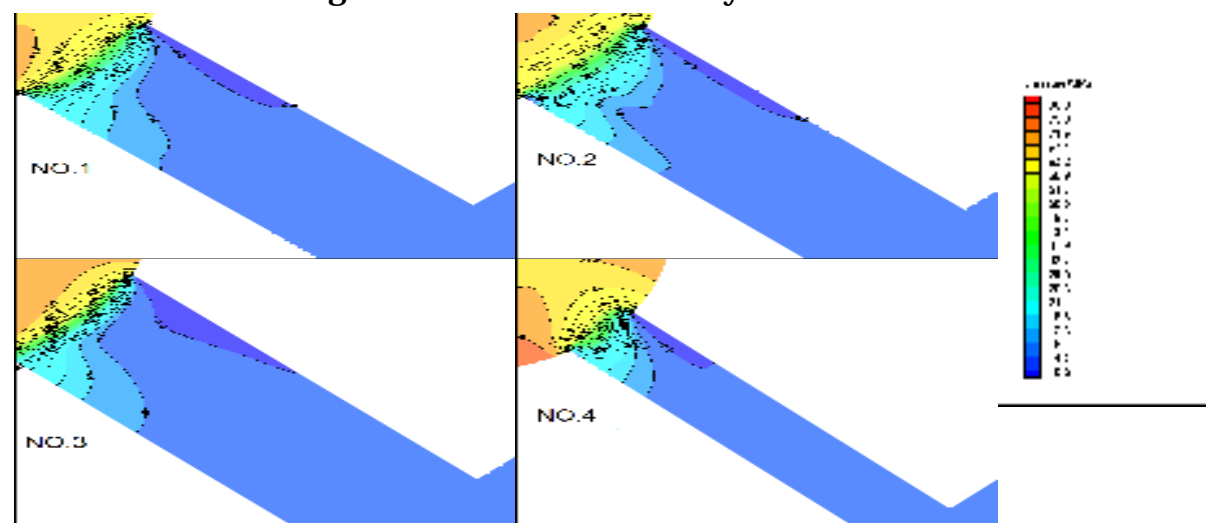

Fig. 9 Oval nozzle pressure distribution

For elliptical nozzles and it's needle valve lift is $0.25 \mathrm{~mm}$, different elliptical short axis length and short axis placement position were simulated, and the phenomenon of hole flow in elliptical nozzle was analyzed. It can be seen from Fig. 7 that in the case of the same long axis, the cavitation layer in the nozzle increases as the length of the elliptical minor axis increases. No. 2 nozzle has the longest short axis length, and its cavitation layer extends from the inlet to the outlet. On the contrary, the shortest axis of the No.3 nozzle, the cavitation layer is also the thinnest. In the horizontal direction, with the 
increase of the short axis, the shape of the more close to the round, the smaller the impact of the wall, the film is more easy to develop cavitation cavitation, It appears that the turbulence intensity of the flow is caused by cavitation in the No. 3 nozzle in the weakest. Comparison of nozzles 1 and 4, representing the long axis vertical and long axis horizontal. The reason is that when the fuel into the nozzle, the first entry from the top of the entrance, for the long axis of the nozzle placed vertically, the fuel deflection angle is small, the blocking effect is smaller. So this is less cavitation than any other situation. As shown in Fig. 8 and Fig. 9, the fluid velocity and pressure gradient in the No. 2 nozzle are larger than those of No. 1 and No. 3 nozzles, and the No.3 nozzle is the smallest. Further note that the longer the short axis, the stronger the intensity of fuel turbulence, the more atomized degree. Comparison of No. 1 and No. 4 nozzles, also found that the speed and pressure gradient to be larger in the No. 4 nozzle, which is more prone to cavitation. All in all, the longer the short axis of the elliptical nozzle, the more prone to cavitation. When the long axis of the elliptical nozzle is placed horizontally, cavitation is more likely to occur.

Effects of the Aspect ratio within control valve on flow characteristics
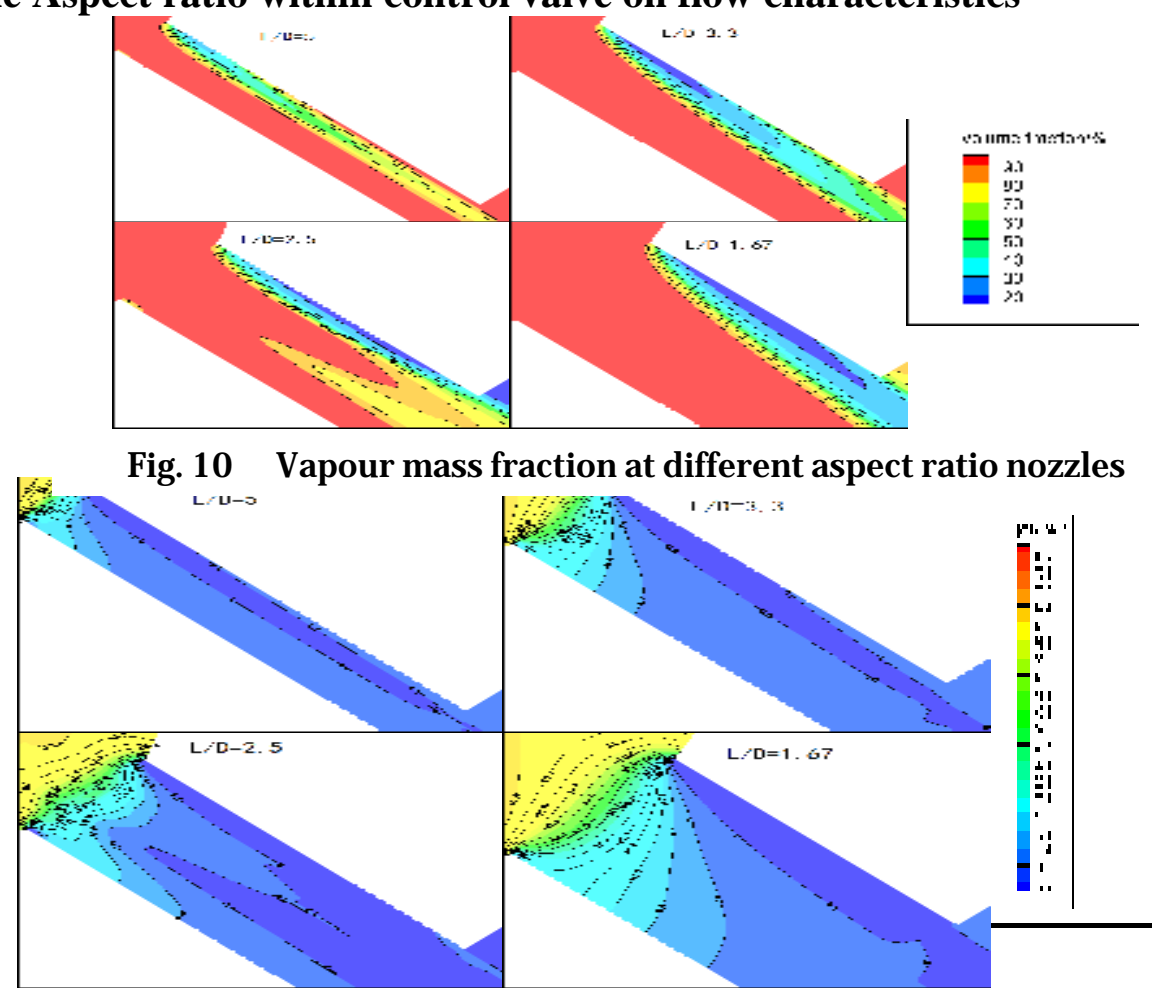

Fig. 11 Pressure distribution at different aspect ratio nozzles

Fig. 10 and Fig. 11 show the cavitation distribution and pressure distribution of the fuel in the nozzle with different aspect ratios. From the simulation results, with the decrease of the aspect ratio, the low pressure area inside the nozzle increases and decreases, and the volume fraction of the steam increases first and then decreases. In the four cases, when the aspect ratio is 2.5 , the fuel turbulence intensity is the strongest and the atomization effect is the best.

\section{Effects of the Tilt angle within control valve on flow characteristics}

Figure 12 and Figure 13 show the cavitation and pressure distributions at different tilt angles $\left(120^{\circ}\right.$, $135^{\circ}, 150^{\circ}$ ). When the inclination angle is $120^{\circ}$, the steam volume fraction is the largest. that is, the cavitation is the most serious. Because the smaller the tilt angle, the greater the angle of the fuel into the nozzle, the stronger the turbulence. There are pressure distribution in Figure 13 can also see that the large angle nozzle compared to the small angle nozzle, the hole at the entrance of the pressure drop will be slightly reduced. When the inclination angle is small, the cavitation in the nozzle becomes more serious. All in all, the small angle of the nozzle is more conducive to fuel atomization. 

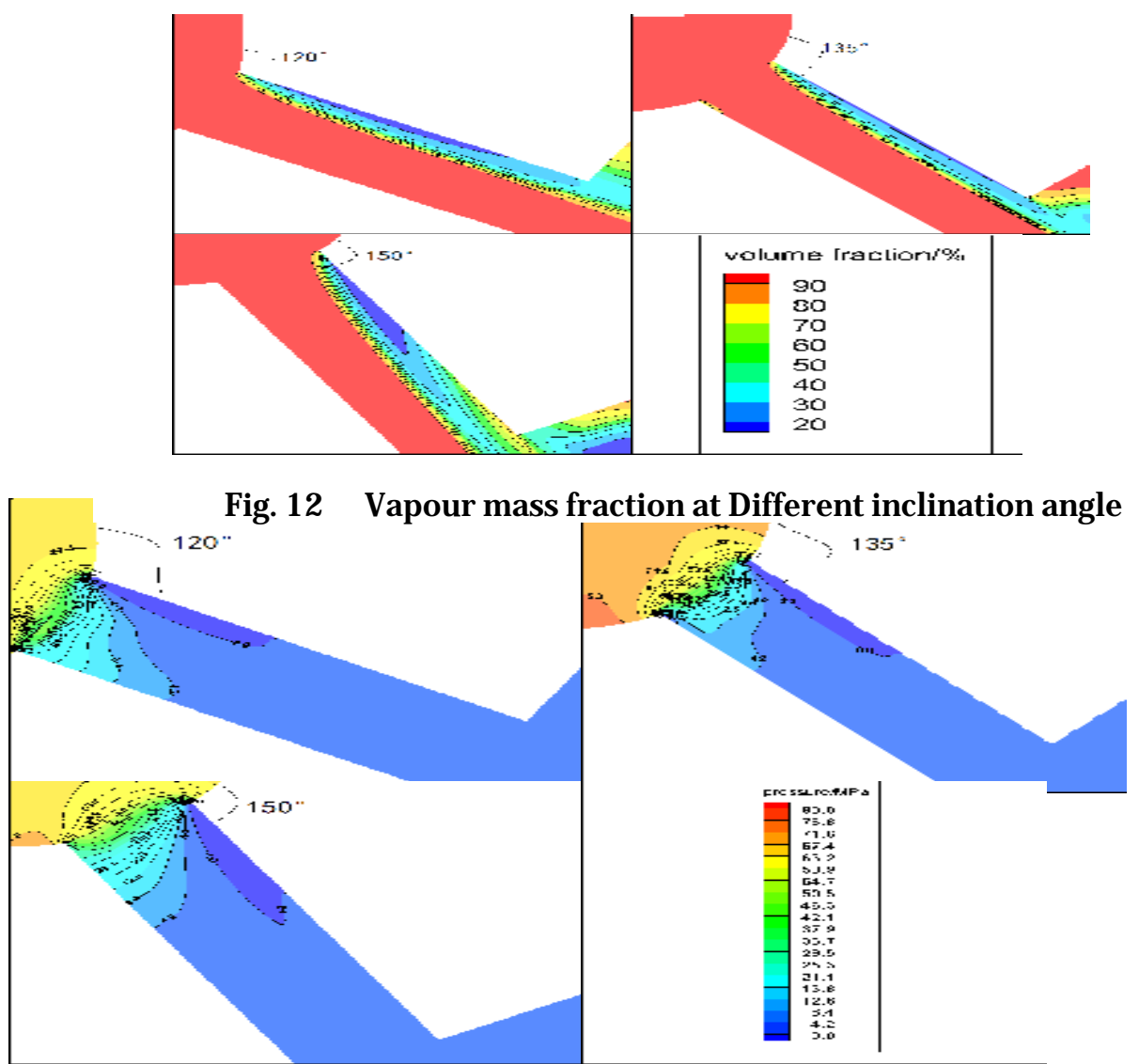

Fig. 13 Pressure distribution at different inclination angle

\section{Conclusion}

Within the present investigation, the nozzle's geometry parameters (including the use of innovative nozzle construction, elliptical geometry nozzle hole, the ratio of nozzle's length to orifice's diameter and hole inclination angle) on the flow and cavitation characteristics within nozzle have been studied by numerical simulations. The main conclusions are listed in the following:

(1) In terms of the three nozzle structures in the text, the cavitation of fuel in medium expansion nozzles is easier to control, and this structure is more conducive to the formation of cavitation. The turbulence intensity of the three-stage tapered nozzle is the weakest, which is not conducive to the cavitation.

(2) For elliptical nozzles, when the elliptical long axis is vertical, the cavitation of the hole is more severe as the length of the minor axis increases. When the long axis of the elliptical nozzle is placed horizontally, the fuel in the nozzle is more prone to cavitation. In the case of circular nozzles with different aspect ratios, with the increase of the aspect ratio, the turbulence intensity of the fuel inside the nozzle increases first and then decreases.

(3) The nozzle installation tilt angle plays an important role that can not be ignored in changing the cavitation flow within the orifice. With the increase of the inclination angle, the cavitation of the hole is weaker. If the inclination angle of the nozzle is appropriately reduced, it is advantageous to improve the atomization quality.

\section{Acknowledgements}

This work was financially supported by the National Natural Science Foundation of China (51506014), Fundamental Research Funds for the Central Universities (106112016CDJXY145501), Chongqing Research Program of Basic Research and Frontier Technology (cstc2016jcyjA0486), China Postdoctoral Science Foundation (2016M592638) and Chongqing Postdoctoral Research Program (Xm2016073). 


\section{References:}

[1] JIANG Guangjun,ZHANG Yusheng,WEN Hua,et al. Study of the generated density of cavitation inside diesel nozzle using different fuels and nozzles[J] . Energy Conversion and Management,2015,103: 208 -217.

[2] MOHAN B,YANG W,YU W,et al. Numerical simulation on spray characteristics of ether fuels[J]. Energy Procedia,2015,75:919 - 924.

[3] QIU Tao, SONG Xin, LEI Yan, XU Hui. Cavitation Process and Flow Characteristics inside Diesel Injector Nozzle[J]. Trantions of the Chinese Society of Agricultural Machinery,2016,(09):359-365.

[4] Hadi Taghavifar,Shahram Khalilarya. Diesel engine spray characteristics prediction with hybridized artificial neural network optimized by genetic algorithm[J]. Energy,2014,71:656-664.

[5] Sheng Huang, Peng Deng. Visualization research on spray atomization, evaporation and combustion processes of ethanol-diesel blend under LTC conditions[J]. Energy Conversion and Management,2015, 106: 911-920.

[6] He Z X,Zhong W J, Wang Q, et al. An investigation of transient nature of the cavitation flow in injector nozzles[J].APPLIED THERMAL ENGINEERING. 2013.54(1):P.56-64.

[7] Zuo yusun,Guo xiuli,Chuan chen,et al. Numerical investigation on effects of nozzle's geometric parameters on the flow and the cavitation characteristics within injector's nozzle for a high-pressure common-rail DI diesel engine[J]. Energy Conversion and Management,2015, 89: 843-861

[8] WANG Yun. A Study of Fuel Temperature Effects on the Cavitation within a Single-hole Diesel Nozzle[D]. East China University of Science and Technology,2015.

[9] SHAO Zhuang, HE Zhixia, ZHONG Wenjun, CHEN Yuhang, TAO Xicheng. Visualization Experiment on Cavitating Flow of Different Length-Diameter Ratios Using Diesel and Biodiesel in Diesel Engine Nozzles[J]. Transactions of CSICE, 2014, (04): 322-327.

[10]HE Zhixia, BAI Jin, WANG Qian, HUANG Yunlong. Visualization Experiment and Numerical Simulation for Cavitating Flow in a Diesel Injector Nozzle[J]. Trantions of the Chinese Society of Agricultural Machinery,2011,(11):6-9+5.

[11]HE Zhixia, ZHAO Ming, WANG Qian, ZHONG Wenjun, SHAO Zhuang, YOU Weihua. Experimental Research of Cavitating Flow in Diesel Fuel Injector Nozzle under Different Back Pressures[J]. Chinese Internal Combustion Engine Engineering,2014,(06): 20-24.

[12]HE Zhixia, WANG Qian, YUAN Jianping, LI Taode. CFD Analysis for Effect of Injection Pressure Fluctuation on Cavitating Flow in Diesel Engine Nozzle[J]. Chinese Internal Combustion Engine Engineering,2009,(01): 64-68.

[13]JIANG Guangjun, ZHANG Yusheng, Medhat Elkelawy, XIAO Gan. Visualization Experiment of Internal Flow of Nozzle and Spray Construction for Various Fuels[J]. Trantions of the Chinese Society of Agricultural Machinery,2014,(05):22-29.

[14]XUE Rui, ZHANG Miao, XU Zhanjun, LIN Leilei. Comparison and Study on Different Cavitation Models[J]. Northwest Hydropower,2014,(02):85-89.

[15]LIU Houlin, LIU Dongxi, WANG Yong, WU Xianfang, ZHUANG Suguo. Applicative evaluation of three cavitating models on cavitating flow calculation in centrifugal pump[J]. Transactions of the Chinese Society of Agricultural Engineering,2012,(16):54-59. 


\title{
Application of Bryophytes and Glass Pumice in Northern China LID Landscape Design
}

\author{
Danning $\operatorname{Han}^{1, a}$ \\ ${ }^{1}$ Liaocheng University,Liaocheng, China \\ ahandanning@yeah.net
}

Key words: bryophytes; glass pumice; ecological landscape design

\begin{abstract}
Based on analyzing the climatic conditions of northern China, the physiological properties of bryophytes and the features of glass pumice, this article proved the feasibility of using bryophytes and glass pumice for northern landscaping, and put forward application methods of bryophytes and glass pumice in LID landscape design.
\end{abstract}

\section{Introduction}

Due to climate characteristics, economic, low quality of ecological infrastructure construction and other factors, the ecosystem of northern China degenerated seriously. Increasing urban wetlands such as ecological parks, can improve the ecological environment effectively. Therefore, landscape design based on low-impact development (LID) becomes very important. Using bryophytes and glass pumice in northern landscaping as a new method of LID landscape design is worth researching.

\section{The necessity of LID landscape design in northern China}

The decrease of rainwater is adverse to the maintenance of urban wetlands. Take the typical northern cities Jinan and Shijiazhuang as an example, Fig.1 shows that the annual precipitation of these two cities showed obvious decreasing trend in recent years. Based on LID design, urban ecological wetlands could intercept more rainwater than ordinary landscape facilities. Therefore, LID landscape design is very significant in northern China.
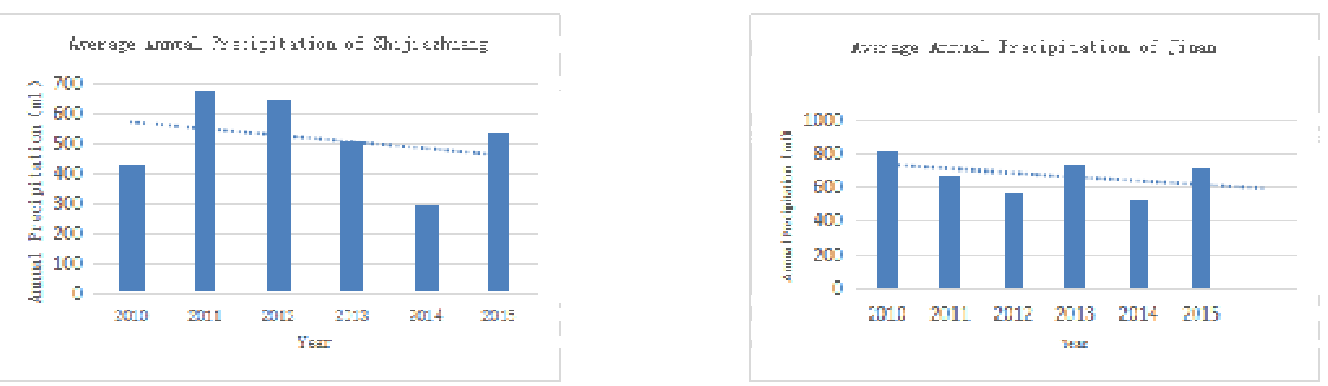

Figure 1 Average Annual Precipitation of Shijiazhuang and Jinan [ml] 


\section{The feasibility of application of bryophytes and glass pumice in LID landscape design of northern China}

\section{Properties and advantages of bryophytes.}

Water holding.Bryophytes are small green plants that they only have stems and leaves, but no real roots and vascular bundles [1].They are usually found as ground cover in the wild. Bryophytes have a strong capability of water holding, the maximum water storage capability can reach 5 times of their own dry weight [2].

Soil conservation.Bryophytes can form biological soil crusts, enhance topsoil stability, reduce wind erosion, water erosion and fertility loss. According to a research, when the bryophyta layer grows thicker, its soil conservation capacity becomes stronger.

Cold and drought endurance. Some of the bryophytes can endure cold and drought. Many species of brophytes grow in the north temperate zone, even frigid zone.An experiment showed that the bryophytes could still grow normally after being baked at high temperature from $40{ }^{\circ} \mathrm{C}$ to $50{ }^{\circ} \mathrm{C}$ [3].

Easy planting and management. Bryophytes require less about substrate, they can absorb moisture and nutrient through rainwater and other non-artificial methods. They can preserve themselves from plant diseases and insect pests. Bryophytes grow fast and have strong capability of acclimatization. According to a research, after some human-caused or forest fires, bryophytes can quickly cover the bare ground and restore the ecological environment [4].

There are many kinds of bryophytes in northern China, for example, there are 62 species of bryophytes in Jinan [5], some of these bryophytes such as Fissidens bryoides, Dicranodontium, Bryum argenteum, can be used for urban landscaping. At present, lawn is commonly used for urban landscaping, but its maintenance cost is high, some maintenance methods may destroy the original ecological balance and cause pollution. The growth conditions of most lawn plants can not fully fit the climate of northern China, however, bryophytes have a strong capability in multiplying and acclimation, and they have not only low planting and maintenance cost, but also good landscaping effect. Therefore, bryophytes can instead some kinds of traditional landscaping plants, and they will have good prospects for application. Glass pumice can be used as a good substrate for bryophytes growth.

\section{Features and advantages of glass pumice}

Glass pumice is made from waste glass. There are many types and colors to choose from. Compared with ceramsite and other water-storage materials, glass pumice has advantages of light weight, high compressive strength, good capacity of water holding and storage, stable physical and chemical properties and so on. Glass pumice is a kind of high quality soil conditioner, and can also be used as a good growth substrate for bryophytes. 
Glass pumice has been widely used as a roof planting substrate, while the roof vegetation is mainly dominated by grass. Using bryophytes instead of grass on impounded roof can increase poundage and reduce cost.

\section{The application of bryophytes and glass pumice in landscape design}

Bryophytes have been used for landscaping in European and American since 1930s. In Japan, bryophytes have been used for landscaping in Saihoji Temple. In China, only in the southern city Hangzhou, a large-scale bryophyte landscape was built in West Lake Park a few years ago. There is still no bryophyte landscape in northern China until now. Here are some suggests on the application of bryophytes and glass pumice in northern landscape design.

Wetlands landscaping. Bryophytes can be used as ground vegetation in the riparian zone, or combine with aquatic plants such as reeds and cattails for ornament. Glass pumice used as substrate can also be used as natural water bank to enrich the landscape gradations. Bryophytes and glass pumice can be designed as ecological floating islands to enrich the waterscape view.

Roof landscaping. Bryophytes and glass pumice can be combined with small flowering shrubs and perennials to decorate pergola or outdoor furniture that on the terraces. In small terraces, bryophytes, glass pumice and sedum can be used to create micro landscape. Bryophytes and their glass pumice substrate can also be used as roof coverings, such as form a figure-ground relation or pattern on the roof.

Low elevation greenbelt landscaping. In recent years, many northern cities have begun to build low elevation greenbelt. Bryophytes and glass pumice can be used in designing dry creek, grass land, waterscape, rainwater garden and other kinds of low elevation greenbelt, or be used to hide the drainage entrances of the low elevation greenbelt. They can also be used for sunken landscape, such as sunken square or dry landscape, etc.

Flower or tree pool design.Bryophytes has the characteristics of shade tolerance and can accept tree crown eluviation, they can absorb nutrients and store the nutrients in the soil, so they can instead of traditional tree pool grates. Combine with glass pumice substrate, they could conserve water and nutrient for soil in the tree pool, and make a better landscape view.

Pavement design. Combine with pebbles, gravel and other materials, bryophytes and glass pumice can be designed as contrasting terrace steps or footpath in different colors and texture of the pavement. Meanwhile this combination can be used to constitute pattern or bedim pavement edge. Bryophytes and glass pumice can also be used to fill the gaps between kerbstones or terrace steps, that could make the pavement edge lines become soft and active to create a natural, irregular visual effect.

Vertical plane decoration. Bryophytes can grow in vertical plane. So bryophytes and glass pumice can be designed as words or pattern to decorate building surface, feature walls, stone tablets and other suitable vertical surface. Glass pumice can also be used with other stone material to make stone gabions. Be planted with bryophytes, these stone gabions could be used as hedge or feature wall to emphasize landscape function, 
like separating space or guiding directions, ect.

\section{Conclusions}

The above analysis shows that the bryophytes have strong adaptability, and the glass pumice can be used as their planting substrate. Using bryophytes and glass pumice for landscaping is beneficial to improve both landscape and ecological effect. Landscape design can be considered from six aspects: wetlands, roof, low elevation greenbelt, flower or tree pool, pavement and vertical plane decoration. These applications have good expectations, and provide an important reference for ecological landscape design based on LID mode in northern China.

\section{References}

[1] Zuntian.Zhao, Tong Cao, in: Flora Bryophytorum Shandong Sinicorum, Shandong science and technology Publishers (2000).

[2] Xinbo Zeng: Study on the Fuction of Siol and Water Conservation of Bryophyta Layer, (Journal of Soil and Water Conservation,China 1995).

[3]Lung Xuezhan, Cao Lintao, Zhou Kunlun: Study on bryophyte:growth habit and its applicability in ecological slope protection, (Environmental Science\& Technology, China 2015).

[4]Smith A J E, in: Bryophyte Ecology, Champan Publishers (1982).

[5] Ren Zhaojie,Huang Zhengli, LI Lin and Zhao Zuntian: Bioindication of bryophytes to environmental quality of Jinan, (Shandong Science, China 2011). 